Public Health Genomics
Public Health Genomics 2017;20:46-57

DOI: $10.1159 / 000452093$
Received: November 20, 2015

Accepted: September 28, 2016

Published online: December 8, 2016

\title{
Community Perceptions of Biobanking Participation: A Qualitative Study among Mexican-Americans in Three Texas Cities
}

\author{
Natalia I. Heredia ${ }^{a}$ Sarah Krasny ${ }^{b}$ Larkin L. Strong ${ }^{c}$ Laura Von Hatten $^{d}$ \\ Lynne Nguyen $^{c}$ Belinda M. Reininger ${ }^{\mathrm{e}}$ Lorna H. McNeill ${ }^{c}$ María E. Fernández ${ }^{a}$ \\ aSchool of Public Health, The University of Texas Health Science Center at Houston, b School of Medicine, Baylor \\ College of Medicine, ' ${ }^{\circ}$ Department of Health Disparities Research, The University of Texas MD Anderson Cancer \\ Center, Houston, TX, dThe University of Texas at Dallas, Dallas, TX, and e School of Public Health, The University of \\ Texas Health Science Center, Brownsville Regional Campus, Brownsville, TX, USA
}

\section{Keywords}

Biobanking · Biospecimens · Biorepository · Qualitative research · Focus groups · Hispanics - Mexican-Americans

\begin{abstract}
Background: Most biospecimens in the US are collected from non-Hispanic Whites, limiting the generalizability of findings. There is a need to increase participation in biobanking among ethnic and racial minorities. The purpose of this study was to use qualitative methods to identify factors that may influence Mexican-American individuals' willingness to participate in biobanking. Methods: We conducted 15 focus groups in three Texas cities with Mexican-American individuals, in both Spanish and English. Results: Lack of knowledge about medical research and biobanks, lack of information about the specifics of biobanking participation, lack of communication of the results, fear of pain or harm, and distrust of the healthcare system or health research were identified as barriers to biobanking participation. Facilitators to participation were altruism, safety, understanding biobanking procedures and purposes, perceived benefits to participation, and culturally appropriate recruitment strategies.
\end{abstract}

\section{KARGER}

(C) 2016 S. Karger AG, Basel

E-Mail karger@karger.com

www.karger.com/phg
Although Mexican-Americans living in Texas are willing to donate biospecimens for altruistic reasons, such as helping society or advancing science, they want more information about what biobanking entails. They want to be assured that participation will not cause them harm and that the research is conducted with good intentions. Conclusion: Results from this study can inform educational materials or interventions to increase Hispanic participation in biobanking.

(c) 2016 S. Karger AG, Basel

\section{Introduction}

Within the last two decades, biobanks have emerged as a driving force in genetic and genomic research, with great potential to enhance the translation of biomedical research into clinical practice and advance personalized medicine. Biobanks are important for identifying the causes and mechanisms of disease, and can contribute to earlier, more accurate prevention, diagnosis, and treatment $[1,2]$. Their usefulness, however, depends on the participation of individuals from diverse racial and ethnic communities to ensure the generalizability of the results
Natalia I. Heredia, MPH

The University of Texas Health Science Center at Houston School of Public Health

7000 Fannin Suite 2576E, Houston, TX 77030 (USA)

E-Mail Natalia.i.heredia@uth.tmc.edu 
Table 1. Focus group composition by site, gender, and language

\begin{tabular}{|c|c|c|c|c|c|c|}
\hline & \multicolumn{2}{|l|}{ Houston } & \multicolumn{2}{|c|}{ Brownsville } & \multicolumn{2}{|l|}{ El Paso } \\
\hline & English & Spanish & English & Spanish & English & Spanish \\
\hline Males & $1(7)$ & $1(7)$ & $1(7)$ & $1(6)$ & $2(10)$ & $2(17)$ \\
\hline Females & $1(8)$ & $1(5)$ & $1(7)$ & $1(15)$ & $1(8)$ & $2(31)$ \\
\hline
\end{tabular}

Number of groups are shown with number of total participants in parentheses.

to the larger community. Inadequate genetic diversity limits the benefits of biobanks, including the promise of personalized medicine for minorities. Although genetic tests may be available for diseases that disproportionately affect minority groups, there is a concern that these tests will not be as applicable to minority groups because the research to develop the tests primarily relied on the genetic material of individuals of European ancestry [3].

Hispanics are underrepresented in biobanks and the resulting research [3-5]. In 2014, 17.4\% of the US population was Hispanic [6]. However, less than $1 \%$ of the specimens collected by seven biorepository facilities across the US and less than $2 \%$ in genome-wide association studies, which often use biobanks as their source for biospecimens, are from Hispanics $[3,4,7]$. Such gross underrepresentation is problematic for generalizability, particularly as the Hispanic population continues to grow in the US [6].

Gauging willingness among Hispanics to participate in biobanking is part of the solution to address this disparity in representation. Research findings generally indicate that Hispanics are less willing to participate in biobanking than non-Hispanic Whites [5, 8]. For example, Hispanics who had previously donated blood were less likely to participate in biospecimen collection than nonHispanic Whites who had previously donated blood [5]. However, since concerns such as fear of lack of protection of medical records and government access to samples are similar for both Hispanics and non-Hispanic Whites [9], we need more research to understand what unique factors may limit participation among Hispanics.

Although studies that include a subset of Hispanic participants have explored the factors that influence biobanking participation $[5,10-14]$, few studies have included an exclusively Hispanic sample [11, 12]. Hispanics note that altruistic reasons, such as benefiting science or society as a whole, play a major role in their decision to donate biospecimens [10, 12-14]. However, Hispanics of different national origins have varying levels of participation in biobanking, indicating a need to examine specific

Community Perceptions of Biobanking Participation subgroups within this heterogeneous population [8]. To our knowledge, only two studies have focused on psychosocial factors that influence biobanking participation among Mexican-Americans. In one study, rural Hispanics, primarily of Mexican-American descent, reported donating biospecimens because of their interest in contributing to science and benefiting future generations [15]. In the other study, Mexican-Americans indicated that lack of knowledge and information about biobanking limits their participation, but that medical distrust was not an important issue for them [16]. The purpose of this study was to assess psychosocial or other factors influencing biobanking participation among MexicanAmericans in Houston, El Paso, and Brownsville, Texas.

\section{Methods}

Design

We conducted a qualitative study using focus group methodology to assess psychosocial factors influencing biobanking participation among Mexican-Americans living in Houston, El Paso, and Brownsville, Texas, USA. We selected these cities because they were part of our National Cancer Institute-funded Community Networks Program, Latinos Contra El Cancer. We conducted 15 focus groups in 2012 and 2013, and analyzed the data in 2013 and 2014. The Institutional Review Board of the University of Texas Health Science Center at Houston reviewed and approved the study. Trained focus group facilitators obtained informed consent from all participants included in the study.

\section{Participant Recruitment}

Research staff recruited Mexican-American adults living in the three Texas cities using convenience sampling techniques. To be eligible, individuals had to be adults over the age of 18 years, speak Spanish or English, be of Mexican origin, live in Houston, El Paso, or Brownsville, and be willing to participate in the focus groups. We posted recruitment flyers at businesses, schools, community centers, apartment complexes, and churches; we also conducted in-person recruitment at these same sites. Flyers included information about the focus groups and a telephone number to call if interested in participating. Community partners assisted with recruitment by distributing flyers and talking with potential participants. Participants were assigned to focus groups based on their 
Table 2. Focus group guide

1. What do you know about biobanking?

a. Probe: what have you heard about it? Can you describe what it is? What do you think it is?

Read the following description:

Biobanking is the process of collecting and storing specimens of human fluids (like blood) and tissues for research. The specimens are often used to develop or test a treatment for diseases such as cancer. Researchers use specimens from people with similar traits for their studies (for example, they might use only blood samples that have been donated by Mexican-American males, age 18-25, who have smoked for 10 years or more). Having specimens from people of different races and ethnicities, different ages, and from men and women helps to ensure that the treatments and tests developed will be appropriate for everybody. There are not as many specimens from Hispanics as there are for other groups.

2. Has anyone ever asked to collect or store your blood or other human tissue for research?

a. Clarify: the sample of blood or other human tissue was not taken by your doctor during a routine visit, but was stored to use in future research projects.

3. How do you feel about people having their blood and other tissue collected and stored for research? And why?

a. If necessary, probe for attitudes: ethical/moral/religious beliefs.

4. How do you feel about the idea of your blood or tissue being stored for a long time?

5. Do you think you would be willing to have your blood or other human tissue collected and stored for research? Why or why not?

6. What things concern or worry you about having your blood or other tissue collected and stored for research?

a. Probe: if they mention fear... What do you think makes collecting blood or other tissue scary?

b. If necessary, probe for potential concerns: fear, misuse of blood/tissue, loss of privacy, mistrust of biobanks, potential for future discrimination, security of the data.

7. What would make you less willing to have your blood or other tissue collected and stored for research?

a. If necessary, probe for potential barriers: lack of information, no direct benefit.

8. What would make you more willing to have your blood or other tissue collected and stored for research?

a. Probe: what things would make you think it is a good idea?

b. If necessary, probe for potential facilitators: provider recommendation, word-of-mouth, materials/information in English and Spanish, bilingual staff, staff from same racial/ethnic group, financial incentives, location and time of blood collection, control over how blood is used and by whom.

9. Do you think it is important for blood and other tissue to be collected for research? Why or why not?

10. What would be some good things about having your blood and other tissue collected and stored for research?

a. If necessary, probe for potential benefits: helping other people, scientific progress, receiving results from blood tests.

11. If you have children, would you consent to having their blood collected and stored for research? Why or why not?

12. How do you think others in your community feel about collecting and storing blood and other tissue for research?

13. Is there anything we didn't discuss about biobanking that you would like to tell us or think we should know?

gender (males and females in separate groups), language (English or Spanish groups), and city of residence (Brownsville, Houston, or El Paso) [17, 18]. Four focus groups were held in Brownsville, seven in El Paso, and four in Houston. Seven groups were conducted in English and eight in Spanish. Eight groups were conducted with men and seven with women. See Table 1 for type of focus group by language, location, and gender.

\section{Development of a Focus Group Guide}

The research team developed a semi-structured, open-ended focus group guide to explore participants' knowledge about biobanking and their perceived barriers and facilitators to future bio- banking participation. The focus group guide was developed following an in-depth review of the literature on minority participation in biobanking research. Questions included a basic inquiry to determine the extent of knowledge related to biomedical research and biobanking specifically. The guide included a short definition of biobanking and proceeded to ask participants about their thoughts on contributing biologic samples to a biobank. Prompts were included to guide the facilitator and stimulate conversation if needed. The focus group guide was first written in English and then translated into Spanish by a bilingual team member. It was then back-translated into English to assess the accuracy of the translation. Table 2 contains the focus group questions in English.
Heredia et al. 
Table 3. Demographics of focus group participants

\begin{tabular}{|c|c|}
\hline \multicolumn{2}{|l|}{ Language } \\
\hline English & $47(36.7)$ \\
\hline Spanish & $81(63.3)$ \\
\hline \multicolumn{2}{|l|}{ Gender } \\
\hline Male & $54(42.2)$ \\
\hline Female & $74(57.8)$ \\
\hline Average age (SD), years & $40(15)$ \\
\hline \multicolumn{2}{|l|}{ Partner status } \\
\hline Single/never married & $47(36.7)$ \\
\hline Married or living with someone & $66(51.6)$ \\
\hline Divorced, separated, or widowed & $14(10.9)$ \\
\hline Missing & $1(0.8)$ \\
\hline \multicolumn{2}{|l|}{ Origin } \\
\hline Mexican & $126(98.4)$ \\
\hline Other Hispanic & $2(1.6)$ \\
\hline \multicolumn{2}{|l|}{ Nativity status } \\
\hline USA & $48(37.5)$ \\
\hline Mexico & $78(60.9)$ \\
\hline Other or missing & $2(1.6)$ \\
\hline Average years living in the US (SD) & $23(14)$ \\
\hline \multicolumn{2}{|l|}{ Education } \\
\hline Some elementary or some middle school & $36(28.1)$ \\
\hline Middle school and some high school & $17(13.3)$ \\
\hline High school & $26(20.3)$ \\
\hline Vocational school & $14(10.9)$ \\
\hline Some college & $22(17.2)$ \\
\hline College or graduate school & $13(10.2)$ \\
\hline \multicolumn{2}{|l|}{ Household income } \\
\hline USD 0-9,999 & $32(25.0)$ \\
\hline USD $10,000-19,999$ & $20(15.6)$ \\
\hline USD 20,000 or more & $34(26.6)$ \\
\hline Unknown, refused to answer, or missing & $42(32.8)$ \\
\hline \multicolumn{2}{|l|}{ Medical insurance } \\
\hline No medical insurance & $85(66.4)$ \\
\hline Medicaid, Medicare, or Veteran's military & $18(14.1)$ \\
\hline Private or managed care & $19(14.8)$ \\
\hline Other or missing & $6(4.7)$ \\
\hline Total & $128(100)$ \\
\hline
\end{tabular}

Values are $n(\%)$ unless otherwise indicated. accuracy. The Spanish-language focus groups were not translated into English to preserve the original nuances in the language; instead, bilingual research team members conducted the analysis.

Data Management and Analysis

The analysis team of three bilingual individuals from the research team, analyzed participant demographic characteristics using SPSS v.20. The analysis team used Atlas.ti v.7.0 (Scientific Software Development, $\mathrm{GmbH}$, Berlin) to code and analyze the focus groups using thematic analysis [19].

The analysis team read through all 15 transcripts and developed ideas for codes. After the analysis team met and discussed the ideas for codes, an initial coding scheme was developed. To test its adequacy and comprehensiveness, the team coded six focus groups (each person coded two transcripts) and then revised the coding scheme to ensure all coders agreed on the full meaning and labeling of each code. Subsequently, team members recoded those original six transcripts. Then each team member went on to code three additional transcripts each, completing the coding of all 15 transcripts. During this round of coding, members of the analysis team made notes of questions or discrepancies. The analysis team met to resolve any discrepancies and answer questions as well as modify the coding scheme and recode as needed. Once the transcripts had been updated to reflect the changes agreed upon, a bilingual independent reviewer read through all of the quotations attached to codes with 12 quotations or less to comment on whether or not they should be uncoded or recoded. The analysis team reviewed those comments and made final decisions on how to adjust the coding scheme and the coding.

Once the coding process was finalized, the analysis team used Atlas.ti's co-occurrence explorer tool to begin to understand how the codes related to one another. The co-occurrence tool allowed the team to cluster codes as part of larger themes, a process that is visually represented as a network. The full research team then reviewed the network and provided feedback and interpretation, and the analysis team revised accordingly. During the analysis phase, the coders began to identify differences across types of focus groups. To further explore similarities and differences across focus groups, the analysis team created groupings of transcripts by gender, location, and language preference. This analysis served as a complement to the network-driven analysis and allowed the team to compare and contrast differences in how participants in different focus groups viewed biobanking.

\section{Results}

Procedure

The focus group facilitators held focus groups at a variety of locations including businesses, schools, community centers, apartment complexes, and churches. Each session lasted between 60 and 90 minutes. Facilitators were all bilingual women. Following a brief introduction to the study, the facilitators collected consent forms, participants filled out a brief demographic questionnaire, and the facilitator continued with the focus group guidelines and questions (Table 2). At the end of the focus group, the facilitator gave participants an informational packet on biobanking and a USD 20 gift card. All focus groups were audio recorded with participant consent. They were then transcribed verbatim by a professional transcription service and the research staff reviewed the transcripts for

Community Perceptions of Biobanking

Participation
Public Health Genomics 2017;20:46-57

DOI: $10.1159 / 000452093$ 


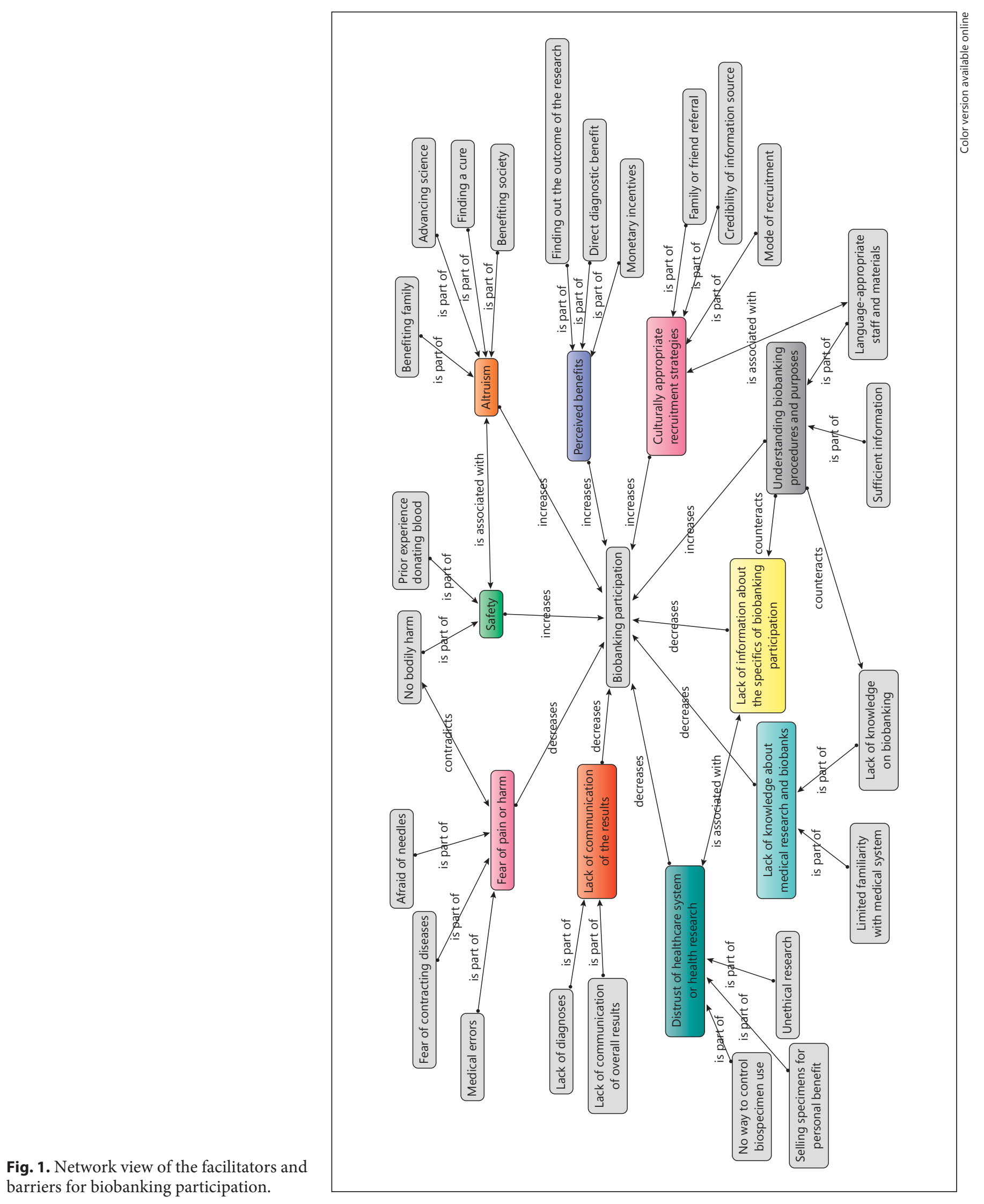

$50 \quad$ Public Health Genomics 2017;20:46-57

Heredia et al. 
We identified barriers and facilitators to biobanking participation. Figure 1 shows the network view of the facilitators and barriers.

\section{Barriers}

Participants noted several barriers to participating in biobanking that included lack of knowledge about medical research generally and biobanking specifically, a lack of information about the specifics of what donating biospecimens entails, lack of communication about the results, distrust of the medical system or providers due to past medical encounters, and fear of pain from needles or harm from contracting diseases.

Lack of Knowledge about Medical Research and

Biobanks

The lack of knowledge about medical research and biobanking included limited familiarity with the medical system and research in general, lack of awareness of biobanking, and confusion between biobanking and other types of donation or medical testing procedures. Participants described potential reasons for limited participation in biobanking among the Mexican-American community. Participants indicated that Mexican-American culture creates a lack of understanding of the medical system, thereby impeding biobanking participation because people are unfamiliar with medical research. Participants from El Paso were most vocal about believing that there is a widespread lack of familiarity with the medical system and research within the Mexican-American community. For example, one participant said: "There's many old people that have never gone to the doctor because they've never felt anything wrong with them. And so they pass that on to the future generations. So they're very closedminded to it." (El Paso, man, English.) Another participant mentioned that the Mexican-American community often thinks that medical research is only conducted with cadavers, and did not realize that a person who is alive can participate.

The majority of participants reported that they had never heard of biobanking or if they had heard the word, they guessed that biobanking meant something else. One participant thought that biobanking was a biopsy, "When they remove a tumor [...] They send it to be checked to make sure that it's not cancerous [...]" (Brownsville, man, Spanish.) Others thought that the blood donated to a biobank could be used to save someone else's life or that an individual could store his or her own blood to be used in the future in case of an illness or injury.

Community Perceptions of Biobanking

Participation
Lack of Information about the Specifics of

Biobanking Participation

In general, participants noted that without clear information of what participation entails, how biospecimens are going to be used, or the impact on one's health, they were not willing to participate. One participant said: "To do something you always have to be informed, for any activity. If you don't have information, if you don't know the risks, if you don't know what's going to happen, how the process is going to be, it is very difficult for someone to want to participate." (El Paso, man, Spanish.) Another participant indicated that a lack of information in the community leads to distrust which could further thwart efforts to recruit members of the Mexican-American community to donate specimens. Although the lack of information about biobanking was apparent in all focus groups, it was most commonly cited as a barrier to participation by male participants from El Paso and Houston.

\section{Lack of Communication of the Results}

Participants expressed that a lack of communication from researchers about both the results of the studies that used their biospecimens as well as their individual personal results was a barrier to participation. A few participants in the female groups in El Paso and Houston were concerned that after donating their biospecimens they would not hear any news on the overall findings of the research to know if they helped others, or whether their donation had resulted in any benefit to the population's health. Only male participants explicitly stated that not receiving individual results (i.e., diagnoses) from research done on their specimen was a barrier to participation because then there would be no personal benefit: "If we were getting the results back I would [donate], but since we're not getting results back I don't think I would." (Houston, man, English.) Overall, a lack of feedback from the researchers on study results or individual diagnoses was a barrier.

Fear of Pain or Harm

The fear of experiencing pain or harm as a result of donating a specimen for biobanking was a commonly mentioned barrier to participation. Fears included being afraid of needles, contracting diseases, or medical errors. Many were fearful of blood draws, needles, and having samples taken from places other than veins such as palms, eyes, or bone. One English-speaking female participant from Houston said, "if they didn't know what they were doing or they were kind of fidgeting or hurting you," that would be painful and a barrier to participating. This sen-

Public Health Genomics 2017;20:46-57 DOI: $10.1159 / 000452093$ 
timent was also expressed by other English-speaking women. The fear of experiencing pain or harm as a result of biobanking was more commonly mentioned by English speakers, Houston participants, and female participants. Concerns about being infected with some disease via contaminated needles came up among Spanish speakers from Houston. Some Spanish-speaking participants assumed that biobanking entailed blood transfusions; as such, they were worried that they would contract a disease such as HIV. Some Spanish-speaking participants were hesitant to engage in biobanking due to a feeling that they would be exposing themselves to unnecessary risks. This fear came from previous experience with or knowledge of medical errors. For example, one female participant said that while living in Mexico she had bone marrow drawn without her consent, a procedure that still causes her pain.

Distrust of the Healthcare System or Health Research

Participants described a number of negative outcomes that could result if they donated biospecimens, such as its sale for the personal benefit of the researcher, unethical research such as cloning, and no control over the biospecimen use in the long-term. Selling specimens for personal benefit was only mentioned in focus groups with Spanish speakers from Houston and El Paso and mostly in female focus groups. Spanish-speaking participants were concerned that biospecimens would be used for the benefit of a few individuals instead of society as a whole. One Spanish-speaking man from El Paso said a barrier would be if "it is not for the benefit of the community, but rather for the benefit of just a few [...]. Because there have always been cases, no?, that they are used in things like that, that is, for personal interests." (El Paso, man, Spanish.)

Spanish speakers from Houston and El Paso believed that donated blood could be sold for a profit or could be part of illegal trafficking. Males and females from both language groups in Brownsville and El Paso worried about whether or not biospecimens could be used for harmful purposes or whether the sample would be stored perpetually without ever being used. A few people in the English-language groups across the sites mentioned cloning as a concern: "As long as they don't do what they do in the movies, like clone you 20 times, I guess it's fine." (El Paso, woman, English.) Only participants from El Paso were concerned that after you donate blood you are no longer in control of what kind of research it will be used for. One man said: “After you donate, there's no way of controlling. You don't have a way of controlling what they're going to do with it." (El Paso, man, English.)

\section{Facilitators}

Participants described several aspects of biobanking that would facilitate their participation. These facilitators included altruistic reasons, such as benefiting society, finding cures, advancing science, and potentially helping their families. Participants mentioned the importance of education and information to make their community more knowledgeable about the existence and the purpose of biobanking and how this process would likely lead to increased participation. Participants described the potential to receive information about a previously undiagnosed disease or condition they may have, the relative safety of participating in biobanking, and culturally sensitive recruitment strategies that would facilitate their participation. The barriers and facilitators that emerged were sometimes the inverse of one another, such that most barriers were identified as facilitators if resolved, occasionally through strategies recommended by participants. For example, while participants described a lack of understanding and information about biobanks as an existing barrier to participation, they noted that acquiring sufficient information on the topic would facilitate their future participation.

\section{Altruism}

Participants from all focus groups regardless of gender, language, or location expressed a willingness to participate in biobanking for several altruistic reasons - a desire to benefit society, benefit family members, advance science, and find cures. The desire to benefit society was the most frequently mentioned facilitator to participation across all groups. For example, one man said: "I think that if you are donating your blood or a piece of your skin, it is to try to help, not to benefit yourself." (Brownsville, man, Spanish.) Participants also felt their participation in biobanking could contribute toward advancing science and finding cures for diseases. One woman said that she would participate "because [donating biospecimens] helps them learn and investigate what medicines we need to cure other illnesses." (Houston, woman, Spanish.) The idea of advancing science in particular was more prominent in the following focus groups: Spanish language, female gender, Brownsville, and Houston.

\section{Safety}

Participants from all focus groups felt that biobanking did not represent a major risk or would not cause bodily harm since it required the donation of specimens such as saliva and blood, specimens that the body is able to "easily replace," and thus did not create long-term bodily
52

Public Health Genomics 2017;20:46-57 DOI: $10.1159 / 000452093$
Heredia et al. 
harm. Some were still fearful of needles or the possibility of contracting a disease. The perception that there was minimal harm emerged particularly among female participants from El Paso and Houston who had previous experience with donating blood, plasma, or cord blood. Some participants seemed to rationalize participation by comparing the altruistic reasons and the minimal risk of bodily harm from biobanking, saying: "I don't really mind I guess just to help out others. They're just going to like try to figure out what they can do better to make everything else work. I guess it's a plus for them. It's not going to affect me." (Houston, man, English.)

Understanding Biobanking Procedures and Purposes Having sufficient information on biobanking, defined as knowing what the biospecimens are going to be used for, where they will be stored, what participation requires (e.g., time commitment, type of specimen, and information collected) and potential side effects, was the second most frequently mentioned facilitator. Sufficient information was a potential solution to the barriers of lack of knowledge about biobanking and lack of information on the specifics of biobanking participation. Mention of sufficient information was similar across male and female focus groups from all locations, but the theme was more frequently mentioned by English speakers.

Participants across the focus groups recommended educating the community about the societal benefits that can emerge from scientific discoveries made through biobanking. Participants felt it was important to address people's misconceptions of what biobanking actually means and what it would require from individual participants. Some participants stated that close-mindedness in the Mexican-American community to medical research could be overcome with increased knowledge and information on these types of studies. Participants suggested having programs or events with the expressed purpose of informing their community on biobanking. Overall, there was a sense that providing information on what the specimen would be used for and how it would benefit others would increase participation.

A number of participants from all focus groups mentioned the need for Spanish-language staff and materials to inform the Hispanic community about biobanking. The ability to communicate in Spanish was key, since some participants anticipated the need to ask questions before agreeing to participate or receive information from others. Participants from Houston and El Paso cautioned that information should be available in Spanish at an appropriate reading level.

Community Perceptions of Biobanking

Participation
Perceived Benefits to Participation

Participants noted several benefits to participating, including finding out if they had certain conditions that they were previously unaware of, the monetary incentives of participating, and finding out the outcome of the research as a whole. There was a common perception among all focus group participants, but particularly those who were Spanish-speaking, that biobanking participation would result in a direct and personal diagnostic or therapeutic benefit. When prompted, participants indicated that receiving the results of a blood test would be a benefit to participating. The following potential benefits were highlighted: receiving lab results from blood work, discovering the presence of a disease, learning how to avoid the onset of a disease, or gaining access to treatment in the case of personal illness. This was especially important for participants who had poor personal health or had family members that suffered from a disease. Two participants from Brownsville (one male and one female) assumed that participation would result in increased access to medical care.

When prompted as to strategies that might increase willingness to participate, participants agreed that monetary incentives would be a benefit of participating; however, this was mainly discussed among English speakers. One participant brought up the story of Henrietta Lacks to emphasize the point that individuals should be compensated for donating biospecimens if their tissues end up being used by the scientific community. One Englishspeaking male from El Paso indicated that even just receiving an acknowledgement of his contribution would be a nice benefit. He said: "[...] feedback to tell you, you know what, we did research. It's helping these people or it's going to help. We can find out this and that. More information to us, to me as the donor [...] At least something to acknowledge you." Some individuals wanted to know the outcome of the research, such as finding out if their sample was part of research that led to new cures or treatments. These kinds of facilitators could be used to overcome the barriers related to lack of communication of the results.

\section{Culturally Appropriate Recruitment Strategies}

Participants were asked about strategies that might influence willingness to participate, such as having materials in Spanish, having bilingual staff, or using word-ofmouth recruitment. Participants expressed that having a well-informed and credible recruiter, using materials that are easy to understand and linguistically appropriate, and offering information orally rather than just on paper

Public Health Genomics 2017;20:46-57 DOI: $10.1159 / 000452093$ 
would all be important. For example, one participant noted that "there are some people that they understand better if you explain to them instead of reading it." (Brownsville, woman, English.) When asked if staff from the same racial/ethnic group would make them more willing to participate, one individual stated that a similar age, but not the race, of the recruiter would be important. Other participants felt that it was important for friends to encourage each other to get involved in biobanking and to spread the message by word-of-mouth. Since most participants did not have a personal physician or physician friends, they felt that physician-led recruitment would be an ineffective strategy since it would be difficult to relate to them. One English-speaking male from Houston went so far as to say, "I don't know too many physicians. They are really like a stranger." Some individuals did mention that the recruiter or the person informing them about the study needs to appear credible for them to want to participate. A few believed that greater interaction at the time of recruitment (e.g., an explanatory video or face-to-face contact with a researcher), rather than just a brochure or an informed consent document, would increase individuals' participation in biobanks.

\section{Discussion}

This qualitative study examined factors that influence Mexican-American individuals' willingness to participate in biobanking using focus groups in community settings in three cities in Texas: Houston, El Paso, and Brownsville. Findings suggest that there is a lack of understanding of biobanking among the Mexican-American population living in Texas. This may stem from a general lack of education about biobanks, but it is compounded by minimal outreach to the community by researchers to explain the intent of biobanks. The primary barriers to future biobanking participation included lack of knowledge about medical research and the existence of biobanks, lack of information on donating biospecimens, lack of communication of the results, fear of pain or harm, and distrust of the healthcare system or health research.

Despite some barriers, participants across all focus groups were generally willing to donate specimens and often cited altruistic reasons for doing so, including to advance science. They noted that they would be willing to participate as long as they had enough information about the research and their personal bodily safety was protected. Other common facilitators included benefiting soci- ety and finding cures. Several recommendations emerged as a result of the focus group discussions that have important implications for recruitment of Hispanics for biobanking, such as purposeful outreach to the Hispanic community using linguistically appropriate materials and culturally sensitive methods to recruit participants.

There were some barriers that were more salient in certain groups. For example, limited familiarity with medical research and the medical system came up more in El Paso focus groups. Fear of pain due to needles generally came up more often with English speakers, Houston residents, and female participants, while the concern about dirty needles and contamination came up mostly with Spanish speakers from Houston. Similarly, the fear that some participants expressed about selling of specimens for the personal benefits of doctors or researchers was mentioned exclusively in the groups with Spanish speakers from Houston and El Paso. Concerns about unethical research were mostly expressed in groups from Brownsville and El Paso.

Females in El Paso and Houston were concerned about not hearing any feedback about the results of the research, while male participants were more concerned about not receiving individual results after donating biospecimens. Females from El Paso and Houston who had previous experience donating other specimens such as blood, mentioned the importance of the perception that biospecimen donation is safe. English speakers from all locations most frequently mentioned the need for sufficient information on the biobanking process and the specifics of biospecimen donation. Despite differences in the main focus of the conversations between the different language, site, and gender groups, most individuals believed that they would be willing to donate if the barriers they experienced were appropriately addressed.

Many of our findings are consistent with those of other studies examining Hispanics' willingness to participate in biobanking. As observed in studies with other Hispanic subgroups, Mexican-Americans in our study expressed an interest in donating biospecimens to a biobank $[8,20]$ and were willing to do so to help advance scientific research or for other altruistic reasons, such as potentially benefiting family in the future [10, 12-16, 21]. Studies with other racial/ethnic minority groups, such as African Americans, Vietnamese and Chinese individuals [10, 13, $14,21]$ as well as non-Hispanic Whites [22], also found that the desire to help future generations and contribute to science was a motivator for participating in biobanking. As in our study, findings from other studies with Mexican-Americans, Spanish-speaking Hispanics, and
54

Public Health Genomics 2017;20:46-57 DOI: $10.1159 / 000452093$
Heredia et al. 
African Americans indicate that the desire to participate in research that leads to new treatments, which may benefit future generations, can outweigh issues of mistrust; they also noted that with the adequate information, the limited mistrust that may exist is not insurmountable $[13,16]$.

Consistent with the findings from our study, studies with other Hispanic subgroups and with other racial/ethnic minority groups identified barriers to participation, which include lack of understanding and awareness about biobanking as well as some distrust in healthcare providers $[10,12-14,21]$. Additionally, fear of pain (e.g., fear of needles) or harm as a result of biobanking participation is a common concern across Hispanics [12, 15, 16] and other racial/ethnic groups [10, 13, 21]. Like in our study, other studies with Puerto Ricans and Mexican-Americans found that the most salient barrier was lack of access to relevant information on biobanking and no awareness of the public benefit of having healthy participants donate biospecimens $[12,16]$. Mexican-Americans in this study, like other minority ethnic/racial communities, have indicated a need for greater public awareness and education on biobanking and genetic research generally [10, 23, 24]. Other researchers have suggested that leveraging social networks would be a helpful way to recruit Hispanics for biobanking participation [11]. The use of social networks is in line with recommendations from our participants who said they would prefer referrals for research from their family and friends rather than from other referral sources such as physicians, with whom they may not have a personal relationship.

Similar to studies across other Hispanics and racial/ ethnic groups [12, 13, 15, 21, 25], some participants had a misperception that participation in biobanking would result in personal benefit such as being diagnosed if they had a disease, and this may also have informed their willingness to participate. Often called diagnostic misconception, this belief is when individuals misunderstand the intent of the research and believe there will be a personal benefit to participating through receipt of additional medical care, such as diagnosis of conditions of which they may have been previously unaware [16]. Researchers have created an instrument that can help assess this diagnostic misconception in the Hispanic community [26], which may be useful for future studies related to Hispanic biobanking participation. If this misperception exists, further education about the intent of participation is warranted.

Although there were many similarities, a few results from our study were not consistent with some of the commonly cited barriers and facilitators previously identified among Hispanics or other racial/ethnic groups. For ex-

Community Perceptions of Biobanking

Participation ample, issues of privacy, consent, and confidentiality have been mentioned consistently as concerns for other populations, including other Hispanic subgroups, other racial/ ethnic minorities, and non-Hispanic Whites $[9,10,13$, $22,27-29]$. Those concerns were not explicitly mentioned as a barrier by our participants. In contrast to other results [21], we did not find that practical or time commitment barriers were particularly important. Our participants mentioned that a barrier to participation is not being informed about the outcomes of the research and if their participation had helped others or advanced science. To our knowledge, this is not a common sentiment explicitly expressed by other groups. Although many of the same barriers and facilitators observed in other racial and ethnic minority groups are applicable to MexicanAmericans, special consideration should be paid to these differences when designing interventions and educational materials for this specific population.

\section{Future Research}

Given the needs vocalized by participants in this study to deliberately reach out to the Hispanic community in a meaningful and appropriate way, researchers and practitioners must look for ways to clearly communicate the purpose of population- and disease-specific biobanking. We should use principles of clear communication [30], such as ensuring appropriate literacy levels and cultural relevancy, to develop educational biobanking materials and interventions to increase participation in the Mexican-American and Hispanic communities. Future studies could use these and other results to improve community engagement [31] and develop or adapt interventions to increase biobanking participation for a Mexican-American population [32,33]. Additionally, future research should focus on designing and implementing interventions for researchers to ensure that they are designing protocols that are responsive to the community's needs and desires, thus making participation less burdensome and more appealing for participants.

\section{Limitations}

This study has some limitations. We assessed future willingness to participate in biobanking and did not speak to individuals who were offered biobanking and refused, or those who have participated in biobanking. We also did not assess the willingness to participate in specific types of research, i.e., diabetes or cancer; answers could have been different for biobanking for specific types of research. The majority (66\%) of our study participants had no insurance and were of low socioeconomic status, limiting applicabil-

Public Health Genomics 2017;20:46-57 DOI: $10.1159 / 000452093$ 
ity of the results across all Mexican-Americans in Texas. Our study design allowed us to discuss differences by gender, location, and language preference, but we were unable to make comparisons by other demographic characteristics, such as insurance status, income, or age. Although we gathered demographic data prior to the focus groups, there is no way to link these data to the qualitative data and thus the participants' comments.

\section{Conclusion}

This study adds insights to the literature on MexicanAmericans' perceptions of biobanking participation. Overall, Mexican-American men and women are willing to participate in biobanking as long as they are provided information about the purpose and use of their biological sample, which has been previously noted in the literature [34]. Given the current low levels of participation by Mexican-Americans in biobanking, coupled with the increasing Hispanic population in the United States, there is a need to quickly address this problem. Results from this study can serve to inform the development or adaptation of interventions and materials to increase awareness in the Mexican-American community, and thus increase their representation in biobanks and the resulting research.

\section{Acknowledgements}

This research was funded through the National Institutes of Health by a Community Networks Program Center grant (U54CA153505), a predoctoral fellowship from the University of Texas Health Science Center at Houston School of Public Health Cancer Education and Career Development Program (R25CA57712), the Clinical and Translational Science Awards (UL1TR000371), and the National Cancer Institute Cancer Center Support Grant (P30 CA016672), as well as through American Cancer Society (MRSG-13-145-01), the Duncan Family Institute through the Center for Community-Engaged Translational Research, and partial funding from the Center for Health Promotion and Prevention Research.

\section{Statement of Ethics}

All procedures performed in studies involving human participants were in accordance with the ethical standards of the institutional and/or national research committee and with the $1964 \mathrm{Hel}-$ sinki declaration and its later amendments or comparable ethical standards.

\section{Disclosure Statement}

The authors have no conflicts of interest to disclose. The content is solely the responsibility of the authors and does not necessarily present the official views of the National Cancer Institute or the National Institutes of Health.

\section{References}

1 Swede H, Stone CL, Norwood AR: National population-based biobanks for genetic research. Genet Med 2007;9:141-149.

2 Henderson GE, Cadigan RJ, Edwards TP, Conlon I, Nelson AG, Evans JP, Davis AM, Zimmer C, Weiner BJ: Characterizing biobank organizations in the US: results from a national survey. Genome Med 2013;5:3.

3 Haga SB: Impact of limited population diversity of genome-wide association studies. Genet Med 2010;12:81-84.

4 Simon MA, Erika E, Bergan R, Norbeck C, McKoy JM, Kulesza P, Dong X, Schink J, Fleisher L: Improving diversity in cancer research trials: the story of the cancer disparities research network. J Cancer Educ 2014;29: 366-374.

5 Scott EA, Schlumpf KS, Mathew SM, Mast AE, Busch MP, Gottschall JL: Biospecimen repositories: are blood donors willing to participate? Transfusion 2010;50:1943-1950.

6 Colby SL, Ortman JM: Projections of the Size and Composition of the US Population: 2014 to 2060: Current Population Reports. Washington, US Census Bureau, 2014, Report No. P25-1143.
7 Kamm L, Bogdanov D, Laur S, Vilo J: A new way to protect privacy in large-scale genomewide association studies. Bioinformatics 2013;29:886-893.

8 Gabriel A, Cohen CC, Sun C: Consent to specimen storage and continuing studies by race and ethnicity: a large dataset analysis using the 2011-2012 national health and nutrition examination survey. ScientificWorldJournal 2014;2014:1-6.

9 Kaufman DJ, Murphy-Bollinger J, Scott J, Hudson KL: Public opinion about the importance of privacy in biobank research. Am J Hum Genet 2009;85:643-654.

10 Dang JH, Rodriguez EM, Luque JS, Erwin DO, Meade CD, Chen MS Jr: Engaging diverse populations about biospecimen donation for cancer research. J Community Genet 2014;5:313-327.

11 Loffredo CA, Luta G, Wallington S, Makgoeng SB, Selsky C, Mandelblatt JS, AdamsCampbell LL; Region 1 Bio-specimen Management of Cancer Health Disparities Program: Knowledge and willingness to provide research biospecimens among foreign-born Latinos using safety-net clinics. J Community Health 2013;38:652-659.
12 Rodriguez EM, Torres ET, Erwin DO: Awareness and interest in biospecimen donation for cancer research: views from gatekeepers and prospective participants in the latino community. J Community Genet 2013;4:461-468.

13 Luque JS, Quinn GP, Montel-Ishino FA, Arevalo M, Bynum SA, Noel-Thomas S, Wells KJ, Gwede CK, Meade CD, Community TB: Formative research on perceptions of biobanking: what community members think. J Cancer Educ 2012;27:91-99.

14 Streicher SA, Sanderson SC, Jabs EW, Diefenbach M, Smirnoff M, Peter I, Horowitz CR, Brenner B, Richardson LD: Reasons for participating and genetic information needs among racially and ethnically diverse biobank participants: a focus group study. J Community Genet 2011;2:153-163.

15 Hohl SD, Gonzalez C, Carosso E, Ibarra G, Thompson B: 'I did it for us and I would do it again': perspectives of rural Latinos on providing biospecimens for research. Am J Public Health 2014;104:911-916. 
16 Ceballos RM, Knerr S, Scott MA, Hohl SD, Malen RC, Vilchis H, Thompson B: Latino beliefs about biomedical research participation a qualitative study on the US-Mexico border. J Empir Res Hum Res Ethics 2014:9: $10-21$.

17 Morgan DL: Focus groups. Ann Rev Sociol 1996;22: 129-152.

18 Kitzinger J, Barbour R (eds): Developing Focus Group Research: Politics, Theory and Practice. London, SAGE, 1999.

19 Braun V, Clarke V: Using thematic analysis in psychology. Qual Res Psychol 2006;3:77-101.

20 Lopez DS, Fernandez ME, Cano MA, Mendez C, Tsai CL, Wetter DW, Strom SS: Association of acculturation, nativity, and years living in the united states with biobanking among individuals of Mexican descent. Cancer Epidemiol Biomarkers Prev 2014;23:402-408.

21 Sanderson SC, Diefenbach MA, Zinberg R, Horowitz CR, Smirnoff M, Zweig M, Streicher S, Jabs EW, Richardson LD: Willingness to participate in genomics research and desire for personal results among underrepresented minority patients: a structured interview study. J Community Genet 2013;4:469-482.

22 Rahm AK, Wrenn M, Carroll NM, Feigelson HS: Biobanking for research: a survey of patient population attitudes and understanding. J Community Genet 2013;4:445-450.
23 Thiel DB, Platt T, Platt J, King SB, Kardia SL: Community perspectives on public health biobanking: an analysis of community meetings on the Michigan biotrust for health. J Community Genet 2014;5:125-138.

24 Lemke A, Wolf W, Hebert-Beirne J, Smith M: Public and biobank participant attitudes toward genetic research participation and data sharing. Public Health Genomics 2010;13: 368-377.

25 Nobile H, Vermeulen E, Thys K, Bergmann MM, Borry P: Why do participants enroll in population biobank studies? A systematic literature review. Expert Rev Mol Diagn 2013;13: 35-47.

26 Knerr S, Ceballos RM: Giving samples or 'getting checked': measuring conflation of observational biospecimen research and clinical care in Latino communities. BMC Med Ethics 2015;16:49.

27 Hoeyer K, Olofsson B-O, Morndal T, Lynoe $\mathrm{N}$ : Informed consent and biobanks: a population-based study of attitudes towards tissue donation for genetic research. Scand J Public Health 2004;32:224-229.

28 Murphy J, Scott J, Kaufman D, Geller G, LeRoy L, Hudson K: Public perspectives on informed consent for biobanking. Am J Public Health 2009;99:2128-2134.

29 Kerath SM, Klein G, Kern M, Shapira I, Witthuhn J, Norohna N, Kline M, Baksh F, Gregersen P, Taioli E: Beliefs and attitudes towards participating in genetic research: a population based cross-sectional study. BMC Public Health 2013;13:114.
30 Koskan A, Arevalo M, Gwede CK, Quinn GP, Noel-Thomas SA, Luque JS, Wells KJ, Meade $\mathrm{CD}$ : Ethics of clear health communication: applying the clean look approach to communicate biobanking information for cancer research. J Health Care Poor Underserved 2012; 23:58-66.

31 Lemke AA, Wu JT, Waudby C, Pulley J, Somkin $\mathrm{CP}$, Trinidad SB: Community engagement in biobanking: experiences from the emerge network. Genomics Soc Policy 2010; 6:1-18.

32 Gao W, Ma GX, Tan Y, Fang C, Weaver J, Jin M, Lai P, Godwin AK: Culturally appropriate education intervention on biospecimen research participation among Chinese Americans. Cancer Epidemiol Biomarkers Prev 2014;23:383-391.

33 Kiviniemi MT, Saad-Harfouche FG, Ciupak GL, Davis W, Moysich K, Hargrave NC, Ambrosone CB, Walker C, Erwin DO: Pilot intervention outcomes of an educational program for biospecimen research participation. J Cancer Educ 2012;28:52-29.

34 Partridge EE: Yes, minority and underserved populations will participate in biospecimen collection. Cancer Epidemiol Biomarkers Prev 2014;23:895-897. 